\title{
Enforced format change to medical education webinar during the coronavirus disease 2019 pandemic
}

\author{
Young-Mee Lee ${ }^{1}$, Hyunmi Park ${ }^{2}$, Sung-Bom Pyun ${ }^{3}$ and Young Wook Yoon ${ }^{4}$ \\ Departments of ${ }^{1}$ Medical Education, ${ }^{2}$ Surgery, ${ }^{3}$ Physical Medicine and Rehabilitation, and ${ }^{4}$ Physiology, Korea \\ University College of Medicine, Seoul, Korea
}

To enhance the teacher's educational competency and promote a positive educational culture, Korea University College of Medicine (KUCM) established Faculty Academy of Medical Education (FAME) in 2012. KUMC has conducted regular seminars 6 times per semester, and it reached to 100 sessions by 2019. It is comprised of 1-hour lectures held at the medical campus with live links to the two other teaching hospitals located 12-hour driving distance. However, since mid-February 2020, the coronavirus disease 2019 (COVID-19) outbreak led to the suspension of all face-to-face gatherings in South Korea, including in house conferences and meetings. Despite the suspension of physical gatherings, KUCM made the decision to persevere with FAME and move the seminars wholly online into webinars. The authors of this paper would like to share what we learned from the medical education webinar, which has been forced to go online due to the COVID-19 pandemic.

The attendance for FAME has not been active, and the main reason for participation was its mandatory once per year requirement for faculty promotion. The mean number of participants per seminar for the 2018/2019 academic year was a lackluster 72 out of 490 full-time faculty members. Hence, when the authors decided to change the seminar format online, we had a gloomy expectation that the participant will be more decreased. However, the conservative expectations were thoroughly shattered by the login attendance number of 210 faculty members on the first webinar on April 8. It was the highest number since its inception in 2012. Interestingly, a higher level of interaction was observed between participants of the other two teaching hospitals and the medical campus using the chat feature during the Q\&A sessions. However, teething technical problems, i.e., disconnection, made us restless during the webinar.

Immediately after the first webinar, an online survey was conducted to get feedback and to correct the technical problems before the second webinar. The survey participants were seventy $33.3 \%$ of the seminar participants). Eighty-three percent of the respondents responded as they could attentively engage the seminar
Received: May 15, 2020 • Revised: May 18, 2020 • Accepted: May 18, 2020 Corresponding Author: Young-Mee Lee (https://orcid.org/0000-0002-4685-9465) Department of Medical Education, Korea University College of Medicine, 73 Goryeodae-ro, Seongbuk-gu, Seoul 02841, Korea

Tel: +82.2.2286.1098 email: ymleehj@korea.ac.kr
Korean J Med Educ 2020 Jun; 32(2): 101-102.

https://doi.org/10.3946/kjme.2020.158

eISSN: 2005-7288

(C) The Korean Society of Medical Education. All rights reserved. This is an open-access article distributed under the terms of the Creative Commons Attribution Non-Commercial License (http:// creativecommons.org/licenses/by-nc/3.0/), which permits unrestricted non-commercial use, distribution, and reproduction in any medium, provided the original work is properly cited. 
even though $78.5 \%$ of them experience some degree of disconnection. Although 37\% of respondents did admit to multi-tasking during the webinar, 56\% of them reported that they could still engage well with the webinar. Eighty-seven percent strongly agreed with the webinar format, with only $5 \%$ disagreeing. Before the second webinar, all possible ways to fix the technical problems were tried including the increased capacity of the internet network and providing practical tips for faculty members for improving better web connection. At the second webinar on April 21, 190 faculty members attended and also showed higher satisfaction scores: mean scores $4.2 \pm 0.7$ of the 5 -point Likert scales.

Surveys from the first two consecutive webinars showed promising results with many positive comments from participants including webinars providing efficiency on time management by eliminating commuting time and space limitation. As with any new technological endeavor, problems with disconnection, buffering, and unfamiliarity using an online platform were identified. The medical school is tackling these issues by securing a more reliable internet network and providing technology education for faculty members.

The webinar format FAME has been successfully launched and has brought new levels of interest and participation from the faculty members. However, the participants' high level of satisfaction may be due to this anomalous situation with many academic conferences cancelled and social distancing increasing people's communication needs. The lack of time and space constraints and being able to multi-task may have increased the webinar attractiveness. It is uncertain whether the current satisfaction levels will be maintained or if it the educational content will translate to its application in the workplace. It is early to gauge the webinar's educational effectiveness, but promising results can be extrapolated from previous studies showing the educational effectiveness of the webinar platform in professional education of healthcare professionals and medical students [1]. Further studies will be conducted on this new platform forced upon us in these tumultuous times, but with the potential to may deliver positive outcomes in the long run.

\section{ORCID:}

Young-Mee Lee: https://orcid.org/0000-0002-4685-9465; Hyunmi Park: https://orcid.org/0000-0002-4134-2213; Sung-Bom Pyun: https://orcid.org/0000-0002-1933-038X; Young Wook Yoon: https://orcid.org/0000-0001-5701-6204 Acknowledgements: The authors would like to show their gratitude to professor Hong-Sik Lee, previous dean of Korea University College of Medicine, who contributed in making FAME a regular faculty development activity. His pivotal first webinar last year was key in the current continuation and success of FAME.

Funding: No funding was obtained for this study.

Conflicts of interest: No potential conflict of interest relevant to this article was reported.

Author contributions: YML designed the webinar for faculty medical education and the online survey, data analysis, and wrote a manuscript. HP contributed to major revision of the manuscript and English translation. SBP and YWY implemented the webinar program and contributed to data collection and writing the manuscript.

\section{References}

1. Gegenfurtner A, Ebner C. Webinars in higher education and professional training: a meta-analysis and systematic review of randomized controlled trials. Educ Res Rev. 2019;28:100293. 